\title{
Schiedsrichterbefangenheit und anwaltliche Versicherungsmandate
}

\author{
Thomas Pfeiffer
}

\section{Einleitung}

Zu den Arbeitsfeldern des Jubilars zählt die Schiedsgerichtsbarkeit. Ein klassisches Thema des Schiedsverfahrensrechts bildet die Sicherung der Unabhängigkeit und Unparteilichkeit von Schiedsrichtern. Ihre große Bedeutung kommt etwa darin zum Ausdruck, dass die IBA dem Thema möglicher Interessenkonflikte von Schiedsrichtern ein eigenes Regelwerk gewidmet hat. ${ }^{1}$ Diese Bedeutung beruht einesteils auf den erheblichen Streitwerten, um die es namentlich in der internationalen Handelsschiedsgerichtsbarkeit häufig geht. Sie ist aber auch Ausdruck der Bestellung parteibenannter Personen zu Schiedsrichtern und des breiteren Tätigkeitsspektrums der Schiedsrichter, die - anders als Berufsrichter - ihr Amt nicht hauptberuflich versehen und deswegen häufiger als diese auch in anderweitigen Beziehungen zu einer Partei stehen können.

Im Vordergrund der Fragestellungen steht insoweit meist die Nähe zur Partei. Allerdings kann sich die Frage einer Nähebeziehung auch bezüglich solcher Personen stellen, die ein indirektes Interesse am Ausgang des Rechtsstreits haben, etwa wenn ein Schiedsrichter die im Unterliegensfall „hinter“ einer Partei stehende Versicherung anwaltlich berät oder vertritt. Nur der Klarstellung halber sei an dieser Stelle angemerkt: Die Fokussierung dieses Beitrags auf das anwaltliche Versicherungsmandat enthält eine allen Darstellungszwecken und der Orientierung am häufigeren Fall geschuldete Vereinfachung; Befangenheitsfragen stellen sich natürlich auch dann, wenn etwa ein professoraler Schiedsrichter für eine Versicherung als Rechtsgutachter tätig ist oder war.

\section{Beziehungen zu einer Person mit indirektem Interesse am Verfahrensausgang}

Beziehungen zwischen einem Schiedsrichter und einer Person mit einem indirekten Interesse an dem Verfahrensausgang sind ein bekannter Gegenstand von Befangenheitsproblemen. Deshalb ist beispielsweise eine geschäftliche Verbundenheit mit dem Ehegatten einer Partei genauso schädlich wie die Beziehung zur Partei selbst. Im Schrifttum wird sogar die Nähe zu bestimmten Zeugen thematisiert. ${ }^{2}$

IBA Guidelines on Conflicts of Interest in International Arbitration v. 22.5.2004, http://www. int-bar.org/images/downloads/guidelines\%20text.pdf, 23.5.2011.

Münch, in: Münchener Kommentar zur Zivilprozessordnung, 3. Aufl. 2008, §1026 ZPO, Rz. 33 sehr weitgehend. 
Versteht man Unabhängigkeit als ein objektives Gebot im Sinne des Fehlens einer Abhängigkeitsbeziehung zur Partei und Unparteilichkeit als eine Geisteshaltung, ${ }^{3}$ dann gibt es zwischen diesen beiden Aspekten der Unbefangenheit zwar bei solchen indirekten Beziehungen Unterschiede: Ein Mangel an unparteilicher Geisteshaltung ist im Falle des Versicherungsmandats denkbar (womit noch nichts dazu gesagt ist, ob die Mandatsbeziehung auch einen Schluss hierauf rechtfertigt); hingegen führt die Mandatsbeziehung zur Versicherung möglicherweise zur Abhängigkeit von dieser, nicht aber von der Partei. Allerdings wird man richtigerweise annehmen müssen, dass sich der objektive Standard der Unabhängigkeit und der subjektive der Unparteilichkeit überschneiden. ${ }^{4}$ Außerdem kann es nicht alleine darauf ankommen, ob der Schiedsrichter unabhängig von den Parteien ist. Maßgebend muss nach dem Sinn der Befangenheitsregeln vielmehr sein, ob der Schiedsrichter über den Streit unabhängig entscheiden kann. Diese Fähigkeit kann auch durch Beziehung zu einem Dritten, der ein Interesse am Ausgang des Verfahrens hat, eingeschränkt sein. Ein Schiedsrichter, der den Belangen eines am Ausgang des Verfahrens interessierten Dritten nicht - wo geboten frei entgegentreten kann, verfügt nicht über die für das Schiedsrichteramt notwendige Unabhängigkeit. ${ }^{5}$ Deshalb ist es jedenfalls nicht von vornherein von der Hand zu weisen, dass die Tätigkeit für eine hinter der Partei stehende und im konkreten Fall einstandspflichtige Versicherung die Ablehnung eines Schiedsrichters rechtfertigt.

Eine wesentliche Stütze findet diese Einschätzung in den Maßgaben der IBA-Guidelines on the Conflict of Interest in International Commercial Arbitration. Dieses Regelwerk kann als Ausdruck eines internationalen Standards und einer verbreiteten sowie - überwiegend, wenngleich möglicherweise nicht in allen Einzelheiten - auch anerkannten Praxis angesehen werden; sie kann als Richtlinie bei der Anwendung der Befangenheitsregeln institutioneller Schiedsorganisationen herangezogen werden, ist aber auch eine Erkenntnisquelle, die bei der Auslegung nationalen Schiedsverfahrensrechts zu berücksichtigen ist. ${ }^{6}$

Standard 7 der IBA-Guidelines sieht nun im Einklang mit den vorstehend entwickelten Maßgaben vor, dass schon die Parteien selbst auch indirekte Beziehungen zu den Schiedsrichtern offenlegen müssen. Auf der Liste der möglichen Befangenheitstatbestände tauchen solche indirekten Beziehungen auf der „Waivable Red List“ unter 2.2 auf. Zu den dort aufgeführten Tatbeständen zählen insbesondere Fälle, in denen ein enger Familienangehöriger (Elternteil, Kind, Ehegatte, Lebensgefährte, Geschwisterteil) des Schiedsrichters enge Beziehungen zu einer Partei unterhält. Genannt wird aber auch die „enge“ Beziehung eines Schiedsrichters zu Personen, bei denen eine Partei im Verlustfalle Regress („recourse“) nehmen kann (Ziffer 2.2.3). Dieselbe Auffassung wurde bereits zuvor von gewichtiger Stimme im Schrifttum formuliert. ${ }^{\text {? }}$

Zwar mag das Merkmal „Recourse“ zunächst eher an Fälle einer Haftungsverantwortung oder eines Anspruchsübergangs denken lassen. Doch wird der Begriff auch im Zusam-

3 Etwa Lionnet/Lionnet, Handbuch der internationalen und nationalen Schiedsgerichtsbarkeit, 3. Aufl. 2005, S. 245 f.

4 Tackaberry/Marriot, Bernstein's Handbook of Arbitration and Dispute Resolution Practice, 4. Aufl. 2003, Band I, S. 684, Rz. 9-108 ff.

5 Treffend etwa Lachmann, Gedanken zur Schiedsrichterablehnung aufgrund Sozietätszugehörigkeit, in Schütze (Hrsg.), Festschrift Geimer, 2002, S. 513, 520.

6 Pfeiffer, Befangenheit von Schiedsrichtern - international gesehen, IDR 2004, 105.

7 Stein/Jonas/Schlosser, Zivilprozessordnung, Band 9, 22. Aufl. 2002, \$1036 ZPO, Rz. 19. 
menhang mit der Inanspruchnahme von Versicherungen verwandt. ${ }^{8}$ Ohnehin kann es auf die Art der Einstandspflicht kaum ankommen, weil die rechtliche Folge (in aller Regel eine Zahlungspflicht) typischerweise dieselbe ist. „Recourse“ wird daher auch Versicherungen umfassen. Freilich verlangen die IBA-Rules nicht nur irgendeine, sondern eine enge Beziehung („,close relationship“) zum regresspflichtigen Dritten, ohne freilich dieses generalklauselartige Merkmal selbst ausdrücklich zu definieren. ${ }^{9}$ Einen gewissen, allerdings auch nicht ohne weiteres übertragbaren Anhaltspunkt bei der Herausarbeitung von Kriterien könnten diejenigen Merkmale bieten, die für die Beziehungen des Anwalts zur Partei selbst von Bedeutung sind: Unterschieden wird zunächst zwischen dem Anwalt selbst auf der einen Seite und Mandaten der Kanzlei auf der anderen Seite (Waivable Red List Ziffern 2.3.1-2.3.4 sowie 2.3.7-2.3.9 einerseits und 2.3.5 sowie 2.3.6 andererseits). Ferner wird unterschieden nach dem wirtschaftlichen Gewicht des Mandats sowie nach der Dauerhaftigkeit des Mandats (vgl. ebenda); abgeschlossene Mandate werden anders behandelt (s. die Tatbestände der „Orange List“ in Ziffer 3). Als weiterer wichtiger Anhaltspunkt ist Standard 6 (b) zu nennen, der anwaltliche Mandate von mit einer Partei konzernverbundenen Unternehmen betrifft. Für diese Fälle betonen die IBA-Guidelines die Vielgestaltigkeit der möglichen Sachverhalte und sehen daher ausdrücklich eine Einzelfallabwägung vor.

Versucht man hieraus eine allgemeine Folgerung zu ziehen, so wird man nach den IBAGuidelines sagen können, dass auch ein indirektes Interesse am Ausgang des Verfahrens geeignet sein kann, Zweifel an der Unabhängigkeit und Unparteilichkeit eines Schiedsrichters zu wecken. Nach dem Maßstab der IBA-Guidelines liegt es deshalb nahe, dass auch Beziehungen eines Schiedsrichters zu einer Versicherung, die hinter einer Partei steht, als Befangenheitsgrund ausreichen können. Wie beim konzernverbundenen Unternehmen wird allerdings auch im Falle des Mandats einer hinter der Partei stehenden Versicherung eine Würdigung verschiedener Umstände geboten sein.

Das entspricht zugleich allgemeinen Grundsätzen. Danach kommt es für die Befangenheit darauf an, ob ein Umstand bei objektiver Betrachtung geeignet ist, Zweifel an der Fähigkeit des Schiedsrichters zur unabhängigen oder unparteilichen Ausübung des Schiedsrichteramts zu begründen. Für solche Zweifel kommt es aber nicht auf die formale Unterscheidungen zwischen unmittelbaren und nur mittelbaren Beziehungen eines Schiedsrichters zu einer Partei oder zum Ausgang des Rechtsstreits an. Vielmehr ist es ein ubiquitäres Phänomen, dass menschliches Verhalten von unmittelbaren und mittelbaren Faktoren beeinflusst werden kann. Ist schon während eines Schiedsverfahrens absehbar, dass eine Partei, namentlich die Schiedsbeklagte, im Unterliegensfall in vollem Umfang die Ersatzleistung einer Versicherung in Anspruch nehmen kann, so trifft diese Versicherung ein etwaiges Unterliegen wirtschaftlich ebenso wie eine Partei.

Freilich kann ein einstandspflichtiger Dritter der Partei auch nicht einschränkungslos gleichgesetzt werden. Zunächst ist nämlich nicht auszuschließen, dass bei einer Partei Folgewirkungen (Reputationsverlust, Gefährdung von Geschäftsbeziehungen) eintreten, die bei einer Versicherung nicht zu besorgen sind. Auch aus einem anderen Grund können ersatzpflichtige Dritte, insbesondere Versicherungen, der Partei nicht gleich stehen. Das gilt namentlich auch für die hier als Nähebeziehung in Frage stehende anwaltliche Mandatsbeziehung. Die Beeinträchtigung der Unbefangenheit des Schiedsrichters im Falle einer Man-

8 Tunc, in: International International encyclopedia of comparative law, Band XI/1, 1982, S. 54, Rz. I-94, Fußn. 309, spricht etwa vom „redress against the insurer".

9 Überblick aber etwa bei MünchKomm/Münch, $\$ 1036$ ZPP, Rz. 32. 
datsbeziehung zur Partei beruht auf drei Gründen. Einmal geht es darum, dass ein Schiedsrichter ein eigenes wirtschaftliches Interesse hat, von der Partei nicht (aus Verärgerung oder Enttäuschung über das Verfahrensergebnis) das Mandat entzogen zu bekommen. Zweitens ist nicht auszuschließen, dass der Schiedsrichter mit der betreffenden Partei aufgrund der Mandatsbeziehung (evt. eher unbewusst) wirtschaftlich sympathisiert. Drittens führt eine laufende Mandatsbeziehung notwendig zu einem häufigen Austausch, so dass sich ein Dritter immer fragen wird, ob hier nicht eine unzulässige ex parte-Kommunikation stattfindet oder ein Sympathievorsprung aus relativ größerer persönlicher Nähe erwächst.

Diese Überlegungen treffen auf ersatzpflichtige Dritte jedenfalls nicht stets in gleicher Weise zu. Hier geht es in der Regel allein um das eigene wirtschaftliche Interesse an einer guten Mandatsbeziehung sowie um eine mögliche Sympathie für die Versicherung. Daraus ergibt sich für aus einer Mandatsbeziehung eines Schiedsrichters zu der Versicherung einer Partei: Die für etwaige Beziehungen eines Schiedsrichters zu einer Partei maßgebenden Grundsätze sind wegen dieser Divergenzen nicht ohne Modifikation übertragbar. Es kommt also darauf an, entsprechende Beurteilungskriterien herauszuarbeiten. Dabei wird, wie stets in dieser Materie, soweit erforderlich, zwischen Kriterien für eine Ablehnung und (der niedrigeren Schwelle) für das Bestehen einer Offenbarungspflicht zu unterscheiden sein.

\section{Kriterien für die Befangenheit von Schiedsrichtern wegen einer Mandatsbeziehung zu der hinter der Partei stehenden Versicherung}

\section{Kenntnis}

„Was ich nicht weiß, macht mich nicht heiß", formuliert treffend der Volksmund. Wovon der Schiedsrichter keine Kenntnis hat, das kann er weder offenbaren noch kann es ihn in seiner Tätigkeit beeinflussen. Notwendige Voraussetzung für das Entstehen einer Offenbarungspflicht des Schiedsrichters bezüglich eines Mandatsverhältnisses zu einer hinter der Partei stehenden Versicherung ist es deshalb, dass der Schiedsrichter Kenntnis davon hat, dass die betreffende Versicherung im Falle des Unterliegens einer bestimmten Partei ersatzpflichtig ist.

Im Normalfall tritt die hinter einer Partei stehende Versicherung allerdings nicht offen zutage. Es ist zudem kein allgemeines Prinzip und keine allgemeine Praxis, dass die betreffende Versicherung bei Fällen mit haftungsrechtlichem Einschlag nach außen hervortritt. An dieser Praxis kann sich die Handhabung der Befangenheitsregeln, insbesondere bezüglich der für die Offenbarungspflichten geltenden Sorgfaltsstandards, ausrichten. Auch ein Rechtsanwalt, der selbst oder dessen Kanzlei häufig Versicherungen vertritt, ist deshalb in derartigen Fällen nicht verpflichtet, von sich aus zu ermitteln, ob die von ihm vertretene Versicherung evt. von einem Regress bedroht ist.

Allerdings kann sich eine entsprechende Kenntnis aus der Vorgeschichte des Streits der Parteien ergeben, etwa wenn die vorgelegte vorprozessuale Korrespondenz die Einstandspflicht einer bestimmten Versicherung, die zugleich Mandantin des Schiedsrichters ist, erkennen lässt. Ebenso liegt es, wenn die betreffende Versicherung während des Verfahrens offenbar wird, etwa weil sie - was nach der Erfahrung des Autors vorkommt - im Verfahren selbst in Erscheinung tritt, wenn beispielsweise die versicherte Partei zur Einbindung einer Haftpflichtversicherung in einen Vergleich ersucht, einem Vertreter dieser Versicherung die Anwesenheit in der mündlichen Verhandlung zu gestatten. 


\section{Auftreten der Versicherung im Verfahren}

Tritt die hinter der Partei stehende Versicherung im Verfahren an der Seite einer Partei in Erscheinung, so kann dies in unterschiedlicher Weise geschehen. Die Schwelle zu einem parteiähnlichen Auftreten wird dann überschritten sein, wenn sich der Vertreter der Versicherung in der mündlichen Verhandlung an etwaigen Rechts- oder Vergleichsgesprächen mit eigenen Wortbeiträgen beteiligt, etwa wenn er auf mögliche Verfahrensrisiken für die Position des Schiedsklägers hinweist. In solchen Fällen bestehen regelmäßig Zweifel, ob ein von dieser Versicherung mandatierter Anwalt solchen Ausführungen frei entgegentreten kann. Deshalb ist in einem solchen Fall in aller Regel nicht nur eine Offenbarungspflicht, sondern - wenn ein entsprechender Sachverhalt bereits eingetreten ist - ein Fall der Befangenheit zu bejahen.

Daraus ergibt sich die Frage, ob es sich anders verhält, wenn der Vertreter der Versicherung an der mündlichen Verhandlung nicht aktiv, sondern als passiver Zuhörer teilnimmt. Auch hier kann das maßgebende Kriterium nur sein, inwiefern der Schiedsrichter hinreichend in der Lage bleibt, sein Schiedsrichteramt auch insoweit frei und unbefangen auszuüben als sich dies zum Nachteil der Versicherung auswirkt. Geht man hiervon aus, so kommt es darauf an, ob sich schon die bloße Anwesenheit auf diese Fähigkeit des Schiedsrichters (potentiell) auswirkt. Das wird man nach der Einsicht, dass das beobachtete Objekt durch die Beobachtung beeinflusst wird, ${ }^{10}$ allerdings annehmen müssen. Schon dies spricht dafür, auch die nur passive Anwesenheit einer Versicherung, die zugleich Mandantin des Schiedsrichters ist, als ausreichenden Grund für eine objektiv fehlende Unbefangenheit anzusehen. Dafür spricht auch, dass eine Unterscheidung zwischen einer „aktiv“ in die Verhandlung eingreifenden und einer nur „passiv“ anwesenden Abgrenzungsfragen in seiner Praktikabilität deshalb zweifelhaft ist, weil sich der Verlauf einer Verhandlung nicht stets vorhersehen lässt und weil die Abgrenzung - etwa in den Fällen vernehmbarer Unterhaltungen oder eines Soufflierens - schwierig werden kann. Es kommt hinzu, dass die Anwesenheit der Versicherung - gleichviel ob "aktiv“" oder "passiv“ - bereits als solche ein erhebliches Interesse derselben am Verfahrensausgang zum Ausdruck bringt. Das ist insofern von Bedeutung als die Regresspflicht eine gewisse Schwelle überschreiten muss, damit der Fall die Versicherung hinreichend interessiert - und nur, wenn dies zu bejahen ist, kann man davon ausgehen, dass die Versicherungsverhältnisse auch den Schiedsrichter beeinflussen können. Geht man also davon aus, dass auch die "passive“ Anwesenheit der Versicherung auf der Seite einer Partei bei einer Schiedsgerichtsverhandlung auf ihr Interesse am Ausgang schließen lässt, so dürfte mehr dafür sprechen, auch in einem solchen Fall in der Regel von einer Befangenheit des mandatierten Schiedsrichters auszugehen.

Für die Offenbarungspflicht des Schiedsrichters hinsichtlich des Mandats gilt dies jedenfalls für praktische Zwecke ohnehin: Da ein Schiedsrichter die Entwicklung der mündlichen Verhandlung nicht einschränkungslos vorhersehen kann, wäre es für ihn - selbst wenn man zwischen aktiver und passiver Anwesenheit unterscheiden wollte - zumindest aus Gründen der Vorsicht ratsam sein, eine Mandatsbeziehung vorab aufzudecken.

10 Ein Beispiel hierfür bildet die nur ganz begrenzte Zulässigkeit von Ton- und Fernsehaufnahmen in Gerichtsverhandlungen ( $\$ 169$ S. 2 GVG), die auch der unbeeinflussten Wahrheits- und Rechtsfindung dient, vgl. BVerfGE 103, 44. 


\section{Ausmaß der Einstandspflicht}

Die Befangenheitsfrage stellt sich jedoch in anderer Weise, wenn die Versicherung nicht durch einen eigenen Vertreter anwesend ist. Zwar könnte man überlegen, ob dieser Fall nicht insofern wie der Fall des anwesenden Vertreters zu beurteilen ist, als auch die Möglichkeit einer nachträglichen Unterrichtung der verpflichteten Versicherung den Schiedsrichter beeinflussen könnte. Andererseits ist in diesem Fall - anders als im Fall der Anwesenheit eines Vertreters der Versicherung - nicht ohne weiteres indiziert, dass der Fall für die Versicherung eine beachtliche Bedeutung hat. Deshalb ist festzuhalten: Die Beachtlichkeit des Verfahrens aus Sicht der Versicherung kann und muss sich in diesem Fall aus anderen Gründen ergeben, etwa aus der Höhe des Streitwerts und der Deckungssumme.

Weitere Unwägbarkeiten können hinzu treten. So kann der Deckungsanteil der Versicherung für den Schiedsrichter unklar sein; ebenso kann sich die Frage des Bestehens einer etwaigen Rückversicherung stellen. Allerdings kommt es für das berührte Interesse der Versicherung wohl nicht auf die prozentuale Quote ihrer Einstandspflicht, sondern auf den Betrag ihrer im Raum stehenden Versicherungsleistung an. Bewegt sich diese - was bei internationalen Schiedsverfahren im Bereich der Handelsschiedsgerichtsbarkeit in der Regel zu erwarten ist - in einer Höhe, die einen aus Versicherungssicht unerheblichen Betrag überschreitet, so ist der Ausgang des Schiedsverfahrens für die Versicherung potentiell von Belang. Auf eine bestimmte prozentuale Quotelung kommt es demgegenüber nicht an. Ist der Verfahrensausgang für die Versicherung erheblich, so kommt eine Befangenheit prinzipiell in Betracht.

Daraus folgt: Ist der Streitwert so erheblich, dass ein erhebliches Interesse der Versicherung am Verfahrensausgang in Betracht kommt, wird man eine Offenbarungspflicht des Schiedsrichters annehmen müssen, auch wenn der Schiedsrichter den Umfang der Einstandspflicht der Versicherung nicht kennen kann. Soweit es darauf ankommt, ist der Umfang der Einstandspflicht im Ablehnungsverfahren aufzuklären. Im Übrigen wird man wohl eine Glaubhaftmachungslast der ablehnenden Partei bejahen müssen. ${ }^{11}$

\section{Versicherung und Rückversicherung}

\section{a) Bestehen einer Rückversicherung}

Das Interesse der Versicherung am Ausgang des Rechtsstreits könnte dadurch belanglos werden, dass die Versicherung rückversichert ist. Auch insofern können sich zudem wiederum Fragen der anteiligen Beteiligung stellen, wenn es sich z.B. um eine Quotenrückversicherung oder die Haftung der Rückversicherung sonst begrenzt ist (z.B. bei der Summenexzedentenversicherung). Bleibt der Erstversicherer für einen erheblichen Teil der Haftungssumme selbst verantwortlich, führt die Rückversicherung nicht dazu, dass dem Erstversicherer seine Einstandspflicht gleichgültig sein kann.

Problematisch ist demgegenüber der Fall einer hundertprozentigen Deckung durch den Rückversicherer. Hier stellt sich die Frage, ob das Bestehen einer solchen Rückversicherung das Eigeninteresse des Versicherers am Verfahrensausgang in einer auf die Befangenheit des Schiedsrichters durchschlagenden Weise einschränkt. Dafür spricht, dass der Versicherer an-

11 MünchKomm/Münch, \$1037 ZPO, Rz. 29. 
gesichts des Bestehens einer hundertprozentigen Rückversicherung für eine etwaige Zahlung voll kompensiert wird. Ob dadurch indessen das wirtschaftliche Eigeninteresse des Versicherers ausgeschlossen wird, ist zweifelhaft, denn der Versicherer wird die Rückversicherung nur dann in Anspruch nehmen wollen, wenn dies unvermeidlich ist. Möglicherweise ergibt sich ein Nachteil auch für künftige Rückversicherungskosten. Zudem wird nicht stets gesichert sein, dass die Rückversicherung zur Deckung verpflichtet ist, namentlich wenn die Versicherung bei zweifelhafter Deckung auch aus Kulanzgründen zahlt. Namentlich wird die Einschaltung der Versicherung in das Schiedsverfahren, wie angesprochen, häufig deren Einbindung in einen Vergleich dienen, so dass der Rückversicherer gerade in diesen Fällen seine Einstandspflicht besonders sorgfältig prüfen wird. In der Regel wird daher das Bestehen einer Rückversicherung das Eigeninteresse der Versicherung am Ausgang des Schiedsverfahrens nicht ausschließen können.

Die Beantwortung all dieser Fragen ist allerdings von den konkreten Umständen abhängig, so dass pauschale Antworten schwer fallen. Das maßgebende Kriterium bleibt indessen sachlich stets dasselbe: Es kommt darauf an, ob der Schiedsrichter davon ausgehen muss, dass die Versicherung am Ausgang des Verfahrens ein erhebliches Eigeninteresse hat.

\section{b) Kenntnis von der Rückversicherung}

Zusätzliche Schwierigkeiten entstehen daraus, dass die gesamte Problematik erneut von der Frage nach dem Kenntnisstand des Schiedsrichters überlagert wird. Allerdings ist das Kenntnisproblem bezüglich der Rückversicherung komplexer, weil sich die Frage stellt, wie sich eine Unkenntnis des Schiedsrichters von dem Bestehen und den Einzelheiten einer Rückversicherung auswirkt.

Nimmt man an, dass es nicht schlechthin ausgeschlossen ist, ein Eigeninteresse der Versicherung infolge des Bestehens einer Rückversicherung zu verneinen, könnte man erwägen, den Schiedsrichter - solange Art und Umfang der Rückversicherung unklar sind - bezüglich des Nichtbestehen eines Eigeninteresses der Versicherung trotz ihrer Leistungspflicht für gleichsam "gutgläubig" zu halten. Mehr dürfte jedoch für die umgekehrte Einschätzung sprechen: Die Leistungspflicht der Versicherung reicht, wenn der Betrag hinreichend erheblich ist, für die Annahme eines Eigeninteresses der Versicherung grundsätzlich aus. Für den von der Versicherung mandatierten Schiedsrichter muss dies - auch ohne Kenntnis von den Einzelheiten eines Rückversicherungsvertrags - Anlass sein, ein etwaiges Mandatsverhältnis zu der betreffenden Versicherung offenzulegen. Das Bestehen eines das Eigeninteresse der Versicherung ausschließenden Rückversicherungsvertrags (soweit überhaupt vorstellbar) ist jedenfalls eine Ausnahme. Sie mag dann in das Verfahren eingebracht und geprüft werden, wenn die Offenlegung tatsächlich von einer Partei zum Anlass einer Schiedsrichterablehnung genommen wird.

\section{c) Mandat durch die Rückversicherung}

Gedanklich kann man die Frage nach einer Befangenheit infolge eines Versicherungsmandats des anwaltlichen Schiedsrichters noch weiterführen, indem man fragt, inwieweit eine Befangenheit durch ein Mandat der hinter der Partei stehenden Rückversicherung ausgelöst wird. Freilich ruft ein solches Gedankenspiel sogleich die Frage nach der Praktikabili- 
tät eines derartigen potentiell infiniten „Befangenheitsregresses“ hervor. Denn irgendwann ist innerhalb einer Regresskette der Haftende von dem ursprünglich haftungsauslösenden Schiedsverfahren soweit entfernt, dass Auswirkungen auf die Unabhängigkeit und Unparteilichkeit des Schiedsrichters kaum mehr zu befürchten sind. Schon mit einer Präsenz des Rückversicherers im Schiedsverfahren wird schwerlich je zu rechnen sein. Zwar kommt es vor, dass eine Rückversicherung sich in die Regulierung durch den Versicherer einschaltet. Gesprächspartner der Rückversicherung ist aber in solchen Fällen typischerweise die Versicherung, nicht der Versicherte. Typischerweise werden die Rückversicherungsverhältnisse deshalb weder im Schiedsverfahren noch sonst für das Schiedsgericht offenbar werden. Für eine Obliegenheit zur Nachfrage besteht kein Anlass. Ist sie ausnahmsweise offenbar, muss es allerdings bei dem dargestellten abstrakten Kriterium bleiben: Es kommt dann darauf an, ob den Umständen nach davon auszugehen ist, dass der Rückversicherer an dem Ausgang des Schiedsverfahrens ein hinreichend konkretes und erhebliches Eigeninteresse hat.

\section{Einzelheiten der Mandatsbeziehung}

Die erkennbare Erheblichkeit des Versicherungsinteresses am Verfahrensausgang (oben 2.4.) allein wird in der Regel ausreichen, um eine Offenbarungspflicht des mandatierten Anwalts zu begründen. Objektiver Anlass für die Besorgnis der Befangenheit besteht aber in der Regel nur dann, wenn damit ein erhebliches Anwaltsinteresse einhergeht. Das wird man bei einer gegenwärtig bestehenden oder kontinuierlichen Mandatsbeziehung des Anwalts bejahen müssen. Frühere, abgeschlossene Mandate sind zwar regelmäßig auch aufzudecken, unterliegen aber - selbst wenn sie zur Partei bestanden, also erst recht im Hinblick auf eine Versicherung - einer großzügigeren Beurteilung. Sie begründen typischerweise keine Befangenheit, soweit sich nicht aus besonderen Umständen (z.B. Häufigkeit) eine nachwirkende Bindung erkennen lässt.

Besteht die Mandatsbeziehung nicht zu dem Schiedsrichter, sondern zu einem anderen Anwalt der Sozietät, so wird schon im Hinblick auf die Parteien zum Teil danach differenziert, ob das Mandat für die Sozietät (und damit auch für den Schiedsrichter) wirtschaftlich bedeutsam ist (IBA-Guidelines - Waivable Red List, Ziffer 2.3.6), wohingegen andere eine solche Differenzierung als sachwidrig und impraktikabel zurückweisen. ${ }^{12}$ Diese Diskussion ließe sich hier weiterführen, sie führt freilich im Hinblick auf Versicherungsmandate zu keiner spezifisch hierfür geltenden Beurteilung, so dass im vorliegenden Rahmen kein zusätzlicher Erkenntnisgewinn zu erwarten ist. Offenbarungspflichtig wird ein solches Mandat aber regelmäßig sein.

12 Lachmann, Festschrift Geimer, S. 521; ders., Handbuch für die Schiedsgerichtspraxis, 3. Aufl. 2008, Rz. 987 ff.; Kreindler/Schäfer/Wolff, Schiedsgerichtsbarkeit, 2006, Rz. 532. 


\section{Fazit und Zusammenfassung}

Die Unparteilichkeit und Unabhängigkeit des Schiedsgerichts lässt sich auch im Zusammenhang mit anwaltlichen Versicherungsmandaten mit den anerkannten allgemeinen Grundsätzen des Befangenheitsrechts gut absichern. Auf dieser Grundlage kann man sich festhalten:

(1) Ist oder wird die deckungspflichtige Versicherung einer Partei erkennbar, so ist ein anwaltlicher Schiedsrichter zur Offenbarung des Bestehens und der Art einer gegenwärtigen oder früheren Mandatsbeziehung zu der Versicherung verpflichtet, sofern der Verfahrensausgang ein erhebliches Interesse der Versicherung berührt.

(2) Hiervon ist stets auszugehen, wenn das Interesse der Versicherung am Ausgang des Schiedsverfahrens durch deren Einschaltung, namentlich im Rahmen von Vergleichsverhandlungen, erkennbar wird. In anderen Fällen kommt es auf die erkennbare (wirtschaftliche) Erheblichkeit des berührten Interesses der Versicherung an. Die Unkenntnis des anwaltlichen Schiedsrichters vom Umfang der Deckungspflicht der Versicherung schließt eine Offenbarungspflicht nicht aus, wenn der Streitwert eine Erheblichkeit als möglich erscheinen lässt.

(3) Das Bestehen einer Rückversicherung schließt ein erhebliches Eigeninteresse des Versicherers am Ausgang des Schiedsverfahrens selbst bei einer hundertprozentigen Rückversicherung in der Regel nicht aus. Ist die Rückversicherungsdeckung nicht erkennbar, so steht dem Eingreifen einer Offenbarungspflicht nicht entgegen, dass eine Rückversicherung bestehen könnte. Für eine Mandatsbeziehung zur Rückversicherung gelten dieselben Grundsätze wie für Versicherung, wobei aber in tatsächlicher Hinsicht zumindest die Erkennbarkeit der Rückversicherungsverhältnissen weitaus seltener gegeben sein wird als diejenige der bestehenden Versicherung.

(4) Eine Nachforschungs- oder Nachfragepflicht zu den Versicherungsverhältnissen trifft den Schiedsrichter nicht.

(5) Während ein erkennbares Versicherungsinteresse am Verfahrensausgang stets eine Offenbarungspflicht des mandatierten Schiedsrichters auslöst, liegt ein objektiver Zweifel an der Unparteilichkeit und Unabhängigkeit des Schiedsrichters in der Regel nur vor, wenn die eigene Mandatsbeziehung erheblich, also typischerweise entweder gegenwärtig oder dauerhaft oder zwar abgeschlossen ist, aber nachwirkende Bindungen bewirkt. 